\title{
Description of nuclear structures in light nuclei with Brueckner-AMD
}

\author{
T. Togashi ${ }^{1, a}$, Yuhei Yamamoto ${ }^{2}$, and K. Katō ${ }^{1}$ \\ 1 Division of Physics, Graduate School of Science, Hokkaido University, Sapporo 060-0810, Japan \\ 2 Department of Cosmosciences, Faculty of Science, Hokkaido University, Sapporo 060-0810, Japan
}

\begin{abstract}
We develop the new antisymmetrized molecular dynamics (AMD) method, Brueckner-AMD, which makes us perform the AMD calculations starting from the realistic nuclear force. In the Brueckner-AMD, the single-particle orbits of AMD can be applied straightforward to the Bethe-Goldstone equation in the Brueckner theory by using the AMD+Hartree-Fock method, and the $G$-matrices are determined with the single-particle energies of AMD self-consistently. In that sense, in this framework, the $G$-matrix in AMD can be solved theoretically without any corrections. We present the applicability of the Brueckner-AMD to describe not only the ground states but also the excited states for some light nuclei, especially the excited $0_{2}^{+}$state in ${ }^{12} \mathrm{C}$ which is not solved sufficiently by the present shell model approaches, starting from the realistic nuclear force.
\end{abstract}

\section{Introduction}

As one of recent major subjects in theoretical nuclear physics, ab initio calculations [1-7] starting from the realistic nuclear forces are developed remarkablely. One hopes to understand the property of nuclei based on the realistic nuclear forces against the background of the progress of the fundamental understanding of nucler bare interactions and the study of unstable nuclei far from the $\beta$-stable line, which are expected to have unknown structures different from stable nuclei. Nowaday, one can obtain the few-body wave functions starting from the bare Hamiltonian by applying many kinds of few-body exact methods [7]. It is difficult to apply such methods directly to heavier nuclei in viewpoint of computatial costs at this stage, therefore, in many of $a b$ initio approaches, the effective interaction is constructed from the realistic interaction by applying, for example, UCOM [4] and so on. As one of remarkable acheivements in ab initio approaches, the quantum Monte Carlo method [1] has presented the appearance of the $\alpha+\alpha$ structure in ${ }^{8} \mathrm{Be}$ starting from the realistic nuclear force. That suggests that one has the ability to discuss cluster structures based on the realistic nuclear forces.

The existence of cluster states to be weakly bound systems of subunits appearing around thresholds is predicted by the Ikeda diagram [8]. The cluster states in several excited states of a light nucleus can be described as the states different from the shell-like structures. Cluster models are able to describe these states successfully. In unstable nuclei, it is expected that shell and cluster structures coexist because their ground states offten exist around the thresholds of decaying particles. As one of the recent development of the cluster model, the antisymmetrized molecular dynamics (AMD) method [9], where both the shell and cluster structures can be described without any assumption of configurations as the results of the energy variation, has succeeded in understanding and predicting the many properties of unstable nuclei. However, the AMD calculations have been performed with phenomenological interactions so far. For the purpose of the fundamental understanding of nuclear structures, we have recently developed new AMD framework, "Brueckner-AMD” [10-12], which makes AMD available to us with realistic nuclear interactions.

In the Brueckner-AMD, the Brueckner theory [13] to be the basic effective interaction theory is combined with the AMD+Hartree-Fock method [14] in AMD. In this framework, the single-particle orbits defined by the AMD+Hartree-Fock method are applied to the BetheGoldstone equation [15] with the realistic interaction to determine the $G$-matrix and the single-particle energies selfconsistently without any corrections. Therefore, in that sense, the $G$-matrix is calculated as the case of not nuclear matter but a finite nucleus in the Brueckner-AMD. Furthermore, the correlation functions [11] generated from the solutions of the Bethe-Goldstion equaiton construct the central, spin-orbit, and tensor interactions in the $G$-matrix explicitly. In this work, we show the applicability of the Brueckner-AMD to describe not only the ground states but also some excited states for some light nuclei, especially the excited $0_{2}^{+}$state in ${ }^{12} \mathrm{C}$ which is indicated as the welldeveloped $3 \alpha$ cluster state [16-20] the present shell model approaches [21] are hardly able to describe, starting from the realistic nucler force. In calculating this $0_{2}^{+}$state in ${ }^{12} \mathrm{C}$, we adopt the prescription with no assumption of the explicit $3 \alpha$ configurations $[14,22]$.

In this paper, we explain the detail of the formulation of the Brueckner-AMD in Sect. 2. We report the applications of the Brueckner-AMD to some light nuclei and dis-

\footnotetext{
a e-mail: toga@nucl.sci.hokudai.ac.jp
} 
cuss their results in Sect. 3, and summarize this paper in Sect. 4.

\section{Formulation}

\subsection{AMD wave function}

The AMD wave function [9] $|\Phi\rangle$ is represented as an antisymmetrized product of single-particle wave functions for $A$ nucleons (a single Slater determinant):

$$
\begin{aligned}
|\Phi\rangle & =\frac{1}{\sqrt{A !}} \operatorname{det}\left|\phi_{j}\right\rangle \equiv \mathcal{A}\left\{\prod_{j}^{A}\left|\phi_{j}\right\rangle\right\} \\
& =\mathcal{A}\left\{\prod_{j}^{A}\left|Z_{j}\right\rangle \cdot\left|\chi_{j}\right\rangle\right\},
\end{aligned}
$$

where $\mathcal{A}$ is the antisymmetrization operator, and the wave fuction of a nucleon, $\left|\phi_{j}\right\rangle$, is divided into a spatial part, $\left|\boldsymbol{Z}_{j}\right\rangle$, and a spin-isospin part, $\left|\chi_{j}\right\rangle$. The spatial wave function $\left|\boldsymbol{Z}_{j}\right\rangle$ has the form of a Gaussian wave packet:

$$
\left\langle\boldsymbol{r} \mid \boldsymbol{Z}_{j}\right\rangle=\left(\frac{2 v}{\pi}\right)^{3 / 4} \exp \left[-v\left(\boldsymbol{r}-\frac{\boldsymbol{Z}_{j}}{\sqrt{v}}\right)^{2}+\frac{\boldsymbol{Z}_{j}^{2}}{2}\right],
$$

where $\boldsymbol{Z}_{j}=\sqrt{v} \boldsymbol{R}_{j}+i \boldsymbol{P}_{j} / \sqrt{v}$. The spin-isospin wave function $\left|\chi_{j}\right\rangle$ is given by $\chi_{j}=p \uparrow, p \downarrow, n \uparrow$, or $n \downarrow$ for simplicity in this work.

\subsection{G-matrix in the Brueckner-AMD}

A realistic nuclear interaction has a strong repulsion at short-range distances. Therefore, it cannot be treated straightforward by the model wave functions such as the AMD wave function. In applying model wave functions, one needs to use effective interactions. The Brueckner theory [13] is a standard theory for deriving an effective interaction based on the realistic nuclear force, $G$-matrix, between nucleons moving on single-particle orbits. In this theory, in order to calculate the $G$-matrix $\hat{G}$, we use the Bethe-Goldstone equation [15]

$$
\hat{G}=\hat{v}_{2}+\hat{v}_{2} \frac{Q}{\varepsilon_{\alpha}+\varepsilon_{\beta}-\left(\hat{t}_{1}+\hat{t}_{2}\right)} \hat{G}
$$

where $\hat{v}_{2}, Q, \varepsilon_{i}$ and $\hat{t}_{i}$ are a realistic two-body interaction, the Pauli projection operator, the single-particle energy, and the kinetic energy operator, respectively.

The Bethe-Goldstone equation denotes an infinite sum of scattering processes of two nucleons in the medium. In this equation, several methods of choosing the energy denominator of the propagator have been proposed. Here, we adopt the "QT $Q$ (gap) choice" [23] where only kinetic energy appears and there is no single-particle potential in intermediate states, as seen in Eq. (3). In the nuclear matter theory, it is known that the convergence of the hole-line expansion in the $Q T Q$ choice is inferior to that in the "continuous choice" [23]. However, at this stage, we check the adequacy of the $Q T Q$ choice in finite nuclei.

In the AMD method, the Brueckner theory can be applied straightforwards to single-particle orbits that are defined by the AMD+Hartree-Fock method [14]. The "Brueckner-AMD" is a framework for the application of the Brueckner theory to AMD. Following the AMD+Hartree-Fock method, we construct an orthonormal basis $\left\{f_{p}\right\}$ for single-particle orbits by diagonalizing the overlap matrix $B_{i j}$ expressed as

$$
B_{i j} \equiv\left\langle\phi_{i} \mid \phi_{j}\right\rangle=\left\langle\boldsymbol{Z}_{i} \mid \boldsymbol{Z}_{j}\right\rangle \cdot\left\langle\chi_{i} \mid \chi_{j}\right\rangle,
$$

and then we have

$$
\begin{gathered}
\sum_{j} B_{i j} \cdot C_{j p}=\mu_{p} \cdot C_{i p}, \quad \sum_{j} C_{j p}^{*} C_{j q}=\delta_{p, q}, \\
\left|f_{p}\right\rangle \equiv \frac{1}{\sqrt{\mu_{p}}} \sum_{j}\left|\phi_{j}\right\rangle C_{j p}=\sum_{j}\left|\phi_{j}\right\rangle \tilde{C}_{j p} .
\end{gathered}
$$

The states $\left|f_{p}\right\rangle$ are satisfied with the orthogonality: $\left\langle f_{p} \mid f_{q}\right\rangle=$ $\delta_{p, q}$. The Hartree-Fock Hamiltonian matrix can be written with $\left\{f_{p}\right\}$ :

$$
h_{p q} \equiv\left\langle f_{p}|\hat{t}| f_{q}\right\rangle+\sum_{r}\left\langle f_{p} f_{r}|\hat{G}| \mathcal{A}\left\{f_{q} f_{r}\right\}\right\rangle .
$$

Then we obtain the single-particle orbit as the solution $\left|\tilde{f}_{\alpha}\right\rangle$ for the following equations:

$$
\begin{aligned}
& \sum_{q} h_{p q} \cdot g_{q \alpha}=\varepsilon_{\alpha} \cdot g_{p \alpha}, \quad \sum_{q} g_{q \alpha}^{*} g_{q \beta}=\delta_{\alpha, \beta}, \\
&\left|\tilde{f}_{\alpha}\right\rangle \equiv \sum_{q} g_{q \alpha}\left|f_{q}\right\rangle=\sum_{j}\left(\sum_{q} \tilde{C}_{j q} \cdot g_{q \alpha}\right)\left|\phi_{j}\right\rangle \\
&=\sum_{j} \tilde{g}_{j \alpha}\left|\phi_{j}\right\rangle .
\end{aligned}
$$

The single-particle orbits $\left|\tilde{f}_{\alpha}\right\rangle$ are also satisfied with the orthogonality: $\left\langle\tilde{f}_{\alpha} \mid \tilde{f}_{\beta}\right\rangle=\delta_{\alpha, \beta}$. The single-particle energy is obtained as

$$
\varepsilon_{\alpha}=\left\langle\tilde{f}_{\alpha}|\hat{t}| \tilde{f}_{\alpha}\right\rangle+\sum_{\gamma}\left\langle\tilde{f}_{\alpha} \tilde{f}_{\gamma}|\hat{G}| \mathcal{A}\left\{\tilde{f}_{\alpha} \tilde{f}_{\gamma}\right\}\right\rangle .
$$

In this theory, both the single-particle energies $\varepsilon_{\alpha}$ and the $G$-matrix $\hat{G}$ are determined self-consistently. That means that the $G$-matrix within this framework can be determined theoretically without any corrections.

The Pauli projection operator $Q=1-P$ in the BetheGoldstone equation, Eq. (3), where $P$ is the projection operator for occupied states, $P=\sum_{\alpha<\beta}\left|\tilde{f}_{\alpha} \tilde{f}_{\beta}\right\rangle\left\langle\tilde{f}_{\alpha} \tilde{f}_{\beta}\right|$, is introduced in order to take account of the Pauli principle in the scattering processes in the medium. In the nuclear matter, the occupied states are represented as the Fermi gas states below the Fermi momentum $k_{F}$ and the operator $Q$ can be defined in terms of $k_{F}$. In the Brueckner-AMD, the 
occupied states are composed of single-particle orbits with AMD single-particle wave functions, and so it is hard to solve the Bethe-Goldstone directly because of treating the $Q$-operator. Therefore, following the prescription formulated by Bandō et al. [24], which presents an appropriate means of treating Eq. (3) in the finite nuclear systems, we calculate the $G$-matrix in the following two steps: First, ignoring the $Q$-operator $(Q \rightarrow 1)$, we solve the equation for $\hat{G}^{0}$ :

$$
\hat{G}^{0}=\hat{v}_{2}+\hat{v}_{2} \frac{1}{\varepsilon_{\alpha}+\varepsilon_{\beta}-\left(\hat{t}_{1}+\hat{t}_{2}\right)} \hat{G}^{0} .
$$

Second, we solve the following equation to take into account the $Q$-operator:

$$
\hat{G}=\hat{G}^{0}+\hat{G}^{0} \frac{Q-1}{\varepsilon_{\alpha}+\varepsilon_{\beta}-\left(\hat{t}_{1}+\hat{t}_{2}\right)} \hat{G} .
$$

The explicit form of Eq. (10) becomes the algebraic equation

$$
\begin{aligned}
& \sum_{\alpha<\beta}\left\{\delta_{\gamma_{1}, \alpha} \delta_{\delta_{1}, \beta}+\frac{\left\langle\tilde{f}_{\gamma_{1}} \tilde{f}_{\delta_{1}}\left|\hat{G}^{0}\right| \mathcal{A}\left\{\tilde{f}_{\alpha} \tilde{f}_{\beta}\right\}\right\rangle}{e\left(\gamma_{0} \delta_{0}, \alpha \beta\right)}\right\} \times \\
& \left\langle\tilde{f}_{\alpha} \tilde{f}_{\beta}|\hat{G}| \mathcal{A}\left\{\tilde{f}_{\gamma_{0}} \tilde{f}_{\delta_{0}}\right\}\right\rangle=\left\langle\tilde{f}_{\gamma_{1}} \tilde{f}_{\delta_{1}}\left|\hat{G}^{0}\right| \mathcal{A}\left\{\tilde{f}_{\gamma_{0}} \tilde{f}_{\delta_{0}}\right\}\right\rangle,
\end{aligned}
$$

where we have

$$
e\left(\gamma_{0} \delta_{0}, \alpha \beta\right) \equiv \varepsilon_{\gamma_{0}}+\varepsilon_{\delta_{0}}-\left\langle\tilde{f}_{\alpha}|\hat{t}| \tilde{f}_{\alpha}\right\rangle-\left\langle\tilde{f}_{\beta}|\hat{t}| \tilde{f}_{\beta}\right\rangle
$$

The way how to calculate the matrix elements for $\hat{G}^{0}$ to solve Eq. (11) is explained later.

\subsection{G-matrix calculations with correlation functions}

Another expression of the Bethe-Goldstone equation is

$$
\begin{aligned}
\psi_{k l} & =\varphi_{k l}+\frac{Q}{\varepsilon_{\alpha}+\varepsilon_{\beta}-\left(\hat{t}_{1}+\hat{t}_{2}\right)} \hat{G} \cdot \varphi_{k l} \\
& =\varphi_{k l}+\frac{Q}{\varepsilon_{\alpha}+\varepsilon_{\beta}-\left(\hat{t}_{1}+\hat{t}_{2}\right)} \hat{v}_{2} \cdot \psi_{k l},
\end{aligned}
$$

where the following relationship is used:

$$
\left\langle\varphi_{i j}|\hat{G}| \varphi_{k l}\right\rangle=\left\langle\varphi_{i j}\left|\hat{v}_{2}\right| \psi_{k l}\right\rangle .
$$

In the above equations, $\psi_{k l}$ and $\varphi_{k l}$ represent the solution of the Bethe-Goldstone equation and the two-body part of the model wave function, respectively. As an analogy of Eq. (13), the equation for $\hat{G}^{0}$, Eq. (9), becomes

$$
\psi_{k l}^{0}=\varphi_{k l}+\frac{1}{\varepsilon_{\alpha}+\varepsilon_{\beta}-\left(\hat{t}_{1}+\hat{t}_{2}\right)} \hat{v}_{2} \cdot \psi_{k l}^{0},
$$

where $\psi_{k l}^{0}$ represents the solution of Eq. (9) and it has

$$
\left\langle\varphi_{i j}\left|\hat{G}^{0}\right| \varphi_{k l}\right\rangle=\left\langle\varphi_{i j}\left|\hat{v}_{2}\right| \psi_{k l}^{0}\right\rangle .
$$

Then, Eq. (15) can be solved as the differential equation for the two-body wave functions:

$$
\begin{array}{r}
{\left[\hat{T}_{r}-\omega\right]\left\{\delta_{l^{\prime} l_{0}} \cdot \varphi_{l_{0} m}\left(r ; \boldsymbol{Z}_{r_{k l}}\right)-\psi_{l^{\prime} l_{0} m}^{0 J S}(r)\right\}} \\
=\sum_{l^{\prime \prime}} V_{l^{\prime} l^{\prime \prime}}^{J S}(r) \psi_{l^{\prime \prime} l_{0} m}^{0 J S}(r),
\end{array}
$$

where $\hat{T}_{r}$ represents the relative kinetic energy operator and $\omega \equiv\left(\varepsilon_{\alpha}+\varepsilon_{\beta}\right)-T_{C}(i j: k l)$. In this case, $\hat{T}_{r}$ can be expressed as

$$
\hat{T}_{r}=-\frac{\hbar^{2}}{M} \frac{1}{r} \frac{d^{2}}{d r^{2}} r+\frac{\hbar^{2}}{M} \frac{l^{\prime}\left(l^{\prime}+1\right)}{r^{2}},
$$

and $T_{C}(i j: k l)$ is the expectation value of the two-body center-of-mass kinetic energy:

$$
T_{C}(i j: k l) \equiv\left\langle\boldsymbol{Z}_{C_{i j}}\left|\hat{T}_{C}\right| \boldsymbol{Z}_{C_{k l}}\right\rangle /\left\langle\boldsymbol{Z}_{C_{i j}} \mid \boldsymbol{Z}_{C_{k l}}\right\rangle
$$

where $\hat{T}_{C}$ represents the two-body center-of-mass kinetic energy operator and $\left|\boldsymbol{Z}_{C_{k l}}\right\rangle$ is the two-body AMD wave function of the center-of-mass part: $\boldsymbol{Z}_{C_{k l}}=\left(\boldsymbol{Z}_{k}+\boldsymbol{Z}_{k}\right) / \sqrt{2}$. Note that the two-body AMD wave function is decomposed into the center-of-mass and relative parts and its relative one can be applied directly to Eq. (15). Hence, in Eq. (17), $\varphi_{l_{0} m}\left(r ; \boldsymbol{Z}_{r_{k l}}\right)$ is the two-body AMD wave function of the relative part: $\boldsymbol{Z}_{r_{k l}}=\left(\boldsymbol{Z}_{k}-\boldsymbol{Z}_{k}\right) / \sqrt{2}$ for the partial wave, which becomes

$$
\begin{gathered}
\left(\frac{2 v_{r}}{\pi}\right)^{3 / 4} \exp \left[-v\left(\boldsymbol{r}-\frac{\boldsymbol{Z}_{r_{k l}}}{\sqrt{v_{r}}}\right)^{2}+\frac{\boldsymbol{Z}_{r_{k l}}^{2}}{2}\right] \\
=\sum_{l_{0} m} \varphi_{l_{0} m}\left(r ; \boldsymbol{Z}_{r_{k l}}\right)\left|l_{0} m\right\rangle, \\
\varphi_{l_{0} m}\left(r ; \boldsymbol{Z}_{r_{k l}}\right)=\left(\frac{2 v_{r}}{\pi}\right)^{3 / 4} \exp \left[-v_{r} r^{2}\right] \times \\
z_{l_{0} m}\left(2 \sqrt{v_{r}} r ; \boldsymbol{Z}_{r_{k l}}\right),
\end{gathered}
$$

where $v_{r}=v / 2$ and the explicit expression of $z_{l m}(r ; \boldsymbol{Z})$ is given in Appendix A in Ref. [11]. $\psi_{l^{\prime} l_{0} m}^{0 J S}(r)$ is the solution of this equation which has dependences on $(i, j, k, l, \alpha, \beta)$ due to $\omega$ and $\varphi_{l_{0} m}\left(r ; \boldsymbol{Z}_{r_{k l}}\right)$, and $V_{l l^{\prime}}^{J S}(r)$ is the potential for the two-body channel of total angular momentum $J$ and total spin $S$ with the transition between the angular momenta $l$ and $l^{\prime}$ in the realistic interaction $\hat{v}_{2}$,

$$
V_{l l^{\prime}}^{J S}(r)=\int_{\text {spin }} \int d \Omega_{\boldsymbol{r}} \mathcal{Y}_{l S J}^{M^{*}}\left(\Omega_{\boldsymbol{r}}\right) \hat{v}_{2} \boldsymbol{y}_{l^{\prime} S J}^{M}\left(\Omega_{\boldsymbol{r}}\right),
$$

where $\mathcal{Y}_{l S J}^{M}\left(\Omega_{\boldsymbol{r}}\right)$ represents the eigenfunction of the twobody total angular momentum.

In the following, we provide another viewpoint of Eq. (13):

$$
\psi_{k l}=\varphi_{k l}+\frac{Q}{\varepsilon_{\alpha}+\varepsilon_{\beta}-\left(\hat{t}_{1}+\hat{t}_{2}\right)} \hat{G} \cdot \varphi_{k l} \equiv \hat{F}_{k l} \cdot \varphi_{k l}
$$


where we call $\hat{F}_{k l}$ the "correlation function" defined schematically as $\hat{F}_{k l}=\psi_{k l} / \varphi_{k l}$. From Eqs. (13) and (22), one can obtain the $G$-matrix as

$$
\left\langle\varphi_{i j}|\hat{G}| \varphi_{k l}\right\rangle=\left\langle\varphi_{i j}\left|\hat{v}_{2} \cdot \hat{F}_{k l}\right| \varphi_{k l}\right\rangle .
$$

The equation for $\hat{G}^{0}$ has the analogy to Eq. (23):

$$
\left\langle\varphi_{i j}\left|\hat{G}^{0}\right| \varphi_{k l}\right\rangle=\left\langle\varphi_{i j}\left|\hat{v}_{2} \cdot \hat{F}_{k l}^{0}\right| \varphi_{k l}\right\rangle,
$$

where the correlation function $\hat{F}^{0} k l$ is defined as $\hat{F}_{k l}^{0} \equiv$ $\psi_{k l}^{0} / \varphi_{k l}$ by using the solution $\psi_{k l}^{0}$ of Eq. (15). Hence, the $\hat{G}^{0}$ for each $J$ and $S$ channel becomes

$$
G_{l_{0} l^{\prime}}^{0 J S}(r)=\sum_{l^{\prime \prime}} V_{l^{\prime} l^{\prime \prime}}^{J S}(r) \cdot F_{k l}^{0} \underset{k \beta\}}{\{\alpha S}\left[\begin{array}{l}
J S \\
l^{\prime \prime} l_{0}
\end{array}\right](r),
$$

where the correlation function is given by $\psi_{l^{\prime} l_{0} m}^{0 J S}(r)$ and $\varphi_{l_{0} m}\left(r ; \boldsymbol{Z}_{r_{k l}}\right)$ in Eq. (17) as

$$
F_{k l}^{0\{\alpha \beta\}}\left[\begin{array}{l}
J S \\
l^{\prime} l_{0}
\end{array}\right](r)=\psi_{l^{\prime} l_{0} m}^{0 J S}(r) / \varphi_{l_{0} m}\left(r ; Z_{r_{k l}}\right) .
$$

In the above equation, the denotation of $\{\alpha \beta\}$ means the dependences on the single-particle energies $\varepsilon_{\alpha, \beta}$ in $\omega$ of Eq. (17). The correlation function of Eq. (26) is approximated as $m=0$ in $\psi_{l^{\prime} l_{0} m}^{0 J S}(r)$ and $\varphi_{l_{0} m}\left(r ; \boldsymbol{Z}_{r_{k l}}\right)$ because we find that it has little dependence on $m$. The $G^{0}{ }_{l_{0} l^{\prime}}(r)$ does not include explcitly the spin-flip or time-reversal terms such as spin-orbit forces, and therefore, we determine the $\hat{G}^{0}$ with explicit non-central operators as follows:

$$
\hat{G}_{l_{0} l^{\prime}}^{0}=\sum_{\lambda=0}^{2} G_{l_{0} l^{\prime}, \lambda}^{0 S}(r)\left(\boldsymbol{R}_{\lambda} \cdot \boldsymbol{S}_{\lambda}\right),
$$

where $\left(\boldsymbol{R}_{\lambda} \cdot \boldsymbol{S}_{\lambda}\right)$ is a scaler product of an orbital tensor $\boldsymbol{R}_{\lambda}$ and a spin tensor $\boldsymbol{S}_{\lambda}$ of rank $\lambda$. The components of $\lambda=0$, 1 and 2 correspond to the central, spin-orbit, tensor terms in a potential respectively. And then, one can utilize the following equation [25]

$$
\begin{aligned}
& G_{l_{0} l^{\prime}, \lambda}^{S}(r)=\frac{(2 \lambda+1)}{\left\langle l_{0}\left\|\boldsymbol{R}_{\lambda}\right\| l^{\prime}\right\rangle\left\langle S\left\|\boldsymbol{S}_{\lambda}\right\| S\right\rangle} \sum_{J}(-1)^{J-l_{0}-S} \times \\
& (2 J+1) W\left(l_{0} l^{\prime} S S ; \lambda J\right) G_{l_{0} l^{\prime}}^{0 J}(r),
\end{aligned}
$$

where $W\left(l_{0} l^{\prime} S S ; \lambda J\right)$ is a Racah coefficient. The $S$ becomes $S=0$ in the spin-singlet channel or $S=1$ in the spintriplet one. In this calculation, the angular momenta $l_{0}$ and $l^{\prime}$ in $G^{0}{ }_{l_{0}}^{J S}(r)$ are taken as the allowed lowest values for each two-body channel: $l_{0}=l^{\prime}=0$ in the singlet-even (1E) and the triplet-even (3E) central force, $l_{0}=l^{\prime}=1$ in all forces of the singlet-odd (1O) and the triplet-odd (3O) channels, $l_{0}=l^{\prime}=2$ in the $3 \mathrm{E}$ spin-orbit force, and $l_{0}=0, l^{\prime}=2$ in the $3 \mathrm{E}$ tensor force. As a result, the $\hat{G}^{0}$ becomes

$$
\hat{G}^{0}=G_{\lambda=0}^{0}(r)+G_{\lambda=1}^{0}(r) \boldsymbol{L} \cdot \boldsymbol{S}+G_{\lambda=2}^{0}(r) S_{12} .
$$

Then the matrix elements for $\hat{G}^{0}$ becomes

$$
\begin{aligned}
& \left\langle\tilde{f}_{\gamma} \tilde{f}_{\delta}\left|\hat{G}^{0}\right| \mathcal{A}\left\{\tilde{f}_{\alpha} \tilde{f}_{\beta}\right\}\right\rangle \\
& =\sum_{i j k l} \tilde{g}_{i \gamma}^{*} \tilde{g}_{j \delta}^{*} \tilde{g}_{k \alpha} \tilde{g}_{l \beta}\left\langle\phi_{i} \phi_{j}\left|\hat{G}^{0}\right| \mathcal{A}\left\{\phi_{k} \phi_{l}\right\}\right\rangle .
\end{aligned}
$$

By using Eq. (30), one can obtain the $G$-matrix element, $\left\langle\tilde{f}_{\gamma} \tilde{f}_{\delta}|\hat{G}| \mathcal{A}\left\{\tilde{f}_{\alpha} \tilde{f}_{\beta}\right\}\right\rangle$, from Eq. (11).

Here, we consider the following ratio:

$$
C_{Q}^{\alpha \beta} \equiv \frac{\left\langle\tilde{f}_{\alpha} \tilde{f}_{\beta}|\hat{G}| \mathcal{A}\left\{\tilde{f}_{\alpha} \tilde{f}_{\beta}\right\}\right\rangle}{\left\langle\tilde{f}_{\alpha} \tilde{f}_{\beta}\left|\hat{G}^{0}\right| \mathcal{A}\left\{\tilde{f}_{\alpha} \tilde{f}_{\beta}\right\}\right\rangle},
$$

which represents the effects of the $Q$-operator not included in $\hat{G}^{0}$. By using the above value $C_{Q}^{\alpha \beta}$, one can obtain the correlation function $\hat{F}_{k l}$ as

$$
F_{k l}^{\{\alpha \beta\}}\left[\begin{array}{l}
J S \\
l^{\prime} l_{0}
\end{array}\right](r)=C_{Q}^{\alpha \beta} \cdot F_{k l}^{0\{\alpha \beta\}}\left[\begin{array}{l}
J S \\
l^{\prime} l_{0}
\end{array}\right](r)
$$

which reproduces the $G$-matrix element. Hence, the $G$-matrix for each $J$ and $S$ channel becomes

$$
G_{l_{0} l^{\prime}}^{J S}(r)=\sum_{l^{\prime \prime}} V_{l^{\prime} l^{\prime \prime}}^{J S}(r) \cdot F_{k l}^{\{\alpha \beta\}}\left[\begin{array}{l}
J S \\
l^{\prime \prime} l_{0}
\end{array}\right](r) .
$$

Applying Eq. (28) with $G_{l_{0} l^{\prime}}^{J S}(r)$ instead of $G^{0}{ }_{l_{0} l^{\prime}}(r)$, the $G$ matrix with non-central operators can be determined as

$$
\hat{G}=G_{\lambda=0}(r)+G_{\lambda=1}(r) \boldsymbol{L} \cdot \boldsymbol{S}+G_{\lambda=2}(r) S_{12} .
$$

As seen in the above discussion, one can obtain the $G$ matrix as the explicit effective interaction by using the correlation function.

\subsection{G-matrix in Multiconfiguration calculations}

In the multiconfiguration calculations where Slater determinants are superposed, one needs to calculation the expection values between different Slater determinants. In the following, we consider to calculate the expection values of the $G$-matrix in such cases. Note that the $G$-matrix elements are given as the matrix elements for the singleparticle orbits $\left\{\tilde{f}_{\alpha}\right\}$, and then we have

$$
\begin{aligned}
& \frac{\left\langle\Phi^{(a)}|\hat{G}| \Phi^{(b)}\right\rangle}{\left\langle\Phi^{(a)} \mid \Phi^{(b)}\right\rangle} \\
= & \frac{1}{2} \sum_{\alpha \beta \gamma \delta} B_{\gamma \alpha}^{(a b)^{-1}} B_{\delta \beta}^{(a b)^{-1}}\left\langle\tilde{f}_{\alpha}^{(a)} \tilde{f}_{\beta}^{(a)}|\hat{G}| \mathcal{A}\left\{\tilde{f}_{\gamma}^{(b)} \tilde{f}_{\delta}^{(b)}\right\}\right\rangle \\
= & \frac{1}{2} \sum_{\alpha \beta \gamma \delta} B_{\gamma \alpha}^{(a b)^{-1}} B_{\delta \beta}^{(a b)^{-1}} \sum_{i j k l} \tilde{g}_{i \alpha}^{(a) *} \tilde{g}_{j \beta}^{(a) *} \tilde{g}_{k \gamma}^{(b)} \tilde{g}_{l \delta}^{(b)} \times \\
& \left\langle\phi_{i}^{(a)} \phi_{j}^{(a)}|\hat{G}| \mathcal{A}\left\{\phi_{k}^{(b)} \phi_{l}^{(b)}\right\}\right\rangle
\end{aligned}
$$

where $(a),(b)$ denote the attribution to the states $\left|\Phi^{(a)}\right\rangle$, $\left|\Phi^{(b)}\right\rangle$, repectively, and

$$
B_{\alpha \beta}^{(a b)} \equiv \sum_{i j} \tilde{g}_{i \alpha}^{(a) *} \tilde{g}_{j \beta}^{(b)}\left\langle\phi_{i}^{(a)} \mid \phi_{j}^{(b)}\right\rangle .
$$

Then we calculate the $G$-matrix element as

$$
\left\langle\phi_{i}^{(a)} \phi_{j}^{(a)}|\hat{G}| \mathcal{A}\left\{\phi_{k}^{(b)} \phi_{l}^{(b)}\right\}\right\rangle \equiv \frac{1}{2}\left[\left\langle\hat{G}^{(a)}\right\rangle^{*}+\left\langle\hat{G}^{(b)}\right\rangle\right],
$$


where $\left\langle\hat{G}^{(a),(b)}\right\rangle \equiv\left\langle\phi_{i}^{(a)} \phi_{j}^{(a)}\left|\hat{G}^{(a),(b)}\right| \mathcal{A}\left\{\phi_{k}^{(b)} \phi_{l}^{(b)}\right\}\right\rangle$, and $\hat{G}^{(a)}, \hat{G}^{(b)}$ represent the $G$-matrices decided by the calculations of $\left\langle\Phi^{(a)}|\hat{G}| \Phi^{(a)}\right\rangle,\left\langle\Phi^{(b)}|\hat{G}| \Phi^{(b)}\right\rangle$, repectively. Eq. (37) means the $G$-matrix between the different states is approximated "adiabatically" [24]. In this work, we present the validity of this approximation from the obtained results.

\subsection{Energy variation in the Brueckner-AMD}

In the AMD method, the wave-packet positions $\boldsymbol{Z}_{j}$ in Eq. (2) are complex variational parameters in the $A$-body wave function. We use the frictional cooling method of molecular dynamics $[9,26]$ to determine $\boldsymbol{Z}_{j}$. The brief expression of frictional cooling equation is given by

$$
\frac{d \boldsymbol{Z}_{j}}{d t}=-\frac{\partial \mathcal{H}}{\partial \boldsymbol{Z}_{j}^{*}}, \quad \frac{d \boldsymbol{Z}_{j}^{*}}{d t}=-\frac{\partial \mathcal{H}}{\partial \boldsymbol{Z}_{j}},
$$

where $\mathcal{H}$ is the expectation value of Hamiltonian $\hat{H}$. In this framework, the Hamiltonian is expressed as

$$
\hat{H}=\sum_{i} \hat{t}_{i}-\hat{T}_{\mathrm{cm}}+\sum_{i<j} \hat{G}_{i j},
$$

where $\hat{t}_{i}, \hat{T}_{\mathrm{cm}}$ and $\hat{G}_{i j}$ represent the operators of the onebody kinetic energy, the center-of-mass kinetic energy of the system, and the $G$-matrix as two-body interactions, respectively.

In Eq. (38), the next positons of $\boldsymbol{Z}_{j}$ in the time evolution are decided as

$$
\boldsymbol{Z}_{j}(t+\Delta t)=\boldsymbol{Z}_{j}(t)-\Delta t \cdot \frac{\partial \mathcal{H}(t)}{\partial \boldsymbol{Z}_{j}^{*}} .
$$

If the expectation value of energy $\mathcal{H}$ converges in this time evolution, we consider that the optimized energy and state are attained. In the Brueckner-AMD, the derivative of $\boldsymbol{Z}_{j}$ in $\mathcal{H}$ cannot be calculated analytically because the $G$-matrix becomes no explicit function of $\boldsymbol{Z}_{j}$. In this work, we calculate this derivative as the difference in $\boldsymbol{Z}_{j}$. However, the $G$-matrix depends on the configuration of the wave-packet positions $\left\{\boldsymbol{Z}_{j}\right\}$ because single-particle orbits and the Pauli operator $Q$ change with the configuration of $\left\{\boldsymbol{Z}_{j}\right\}$. Therefore, we must determine the $G$-matrix self-consistently for each small displaced configuration of $\left\{\boldsymbol{Z}_{j}+\Delta \boldsymbol{Z}_{j}\right\}$.

\section{Applications}

We apply the Brueckner-AMD method to various light nuclei and present the applicability of this method. In this work, we use the Argonne $v 8$ ' (AV8') [27] as the realistic nuclear force and switch off electromagnetic interactions. In this paper, we present the results of ${ }^{4} \mathrm{He},{ }^{7} \mathrm{Li},{ }^{8} \mathrm{Be},{ }^{9} \mathrm{Be}$, and ${ }^{12} \mathrm{C}$.

\subsection{Energy-level scheme calculations with $J^{\pi}$ projection}

In general, since the AMD wave functions are not good quantum states of the spin and parity, $J^{\pi}$, one cannot discuss the energy-level scheme by a single AMD wave function and must project out the AMD wave functions onto the spin and parity eigenstates, which means the superposition of space-reflected and rotated Slater determinants [9].

The parity projection means a linear combination of two Slater determinants:

$$
\left|\Phi^{ \pm}\right\rangle=\frac{1}{\sqrt{2}}(1 \pm \mathcal{P})|\Phi\rangle,
$$

where $\mathcal{P}$ is the space-reflection operator that operates at the spatial coordinates $\left|\boldsymbol{Z}_{j}\right\rangle$ of each nucleon. In projecting out the intrinsic wave functions onto each spin-parity $J^{ \pm}$state, the spin- and parity-projected state $\left|\Phi_{M K}^{J \pm}\right\rangle$ is expressed as

$$
\left|\Phi_{M K}^{J \pm}\right\rangle=\frac{2 J+1}{8 \pi^{2}} \int d \Omega D_{M K}^{J *}(\Omega) \hat{R}(\Omega)\left|\Phi^{ \pm}\right\rangle
$$

where $D_{M K}^{J}(\Omega)$ is the Wigner $D$-function and $\hat{R}(\Omega)$ is the rotational operator that makes the spatial and spin coordinates rotated by the Euler angle $\Omega$. In the above equation, the integration means a linear combination of differential rotated Slater determinants. The energy expectation value of $\left|\Phi_{M K}^{J \pm}\right\rangle$ is given by

$$
\begin{aligned}
& \left\langle\hat{H}_{K^{\prime} K}^{J \pm}\right\rangle \equiv \frac{\left\langle\Phi_{M K^{\prime}}^{J \pm}|\hat{H}| \Phi_{M K}^{J \pm}\right\rangle}{\left\langle\Phi_{M K^{\prime}}^{J \pm} \mid \Phi_{M K}^{J \pm}\right\rangle} \\
& =\frac{\int d \Omega D_{K^{\prime} K^{*}}^{J *}(\Omega)\left\{\left\langle\Phi^{R}|\hat{H}| \Phi\right\rangle \pm\left\langle\Phi^{\mathcal{P R}}|\hat{H}| \Phi\right\rangle\right\}}{\int d \Omega D_{K^{\prime} K}^{J *}(\Omega)\left\{\left\langle\Phi^{R} \mid \Phi\right\rangle \pm\left\langle\Phi^{\mathcal{P R}} \mid \Phi\right\rangle\right\}},
\end{aligned}
$$

where $\left|\Phi^{(\mathcal{P}) R}\right\rangle \equiv(\mathcal{P}) \hat{R}(\Omega)|\Phi\rangle$. In order to calculate the energy expectation value of the $J^{ \pm}$state correctly, we perform the $K$-mixing by diagonalizing the Hamiltonian ma$\operatorname{trix}\left\langle\Phi_{M K}^{J \pm}|\hat{H}| \Phi_{M K^{\prime}}^{J \pm}\right\rangle$ and norm matrix $\left\langle\Phi_{M K}^{J \pm} \mid \Phi_{M K^{\prime}}^{J \pm}\right\rangle$ simultaneously for the quantum numbers of $K$ and $K^{\prime}$.

In these calculations, we determine the intrinsic wave functions $|\Phi\rangle$ by performing the energy variation for the parity-projected states $\left|\Phi^{ \pm}\right\rangle$. Therefore, we can obtain not only the lowest-energy parity solution corresponding to the ground state but also the excited parity solution, whose parity is opposite to that of the lowest-energy state. Projecting out the solutions of the energy variation, $\left|\Phi^{ \pm}\right\rangle$, onto each $J$ state, we calculate the energy levels. In the following, we present the results of ${ }^{4} \mathrm{He},{ }^{7} \mathrm{Li},{ }^{8} \mathrm{Be}$, and ${ }^{9} \mathrm{Be}$ using the above calculational procedure. For the Gaussian width parameter $v$ of the wave packets in Eq. (2), we determine the optimum value, $v=0.222\left(\mathrm{fm}^{-2}\right)$ for ${ }^{4} \mathrm{He}$, and adopt $v=$ $0.208\left(\mathrm{fm}^{-2}\right)$ for other $p$-shell nuclei for simplicity of calculations, which is the optimum value for ${ }^{8} \mathrm{Be}$.

Fig. 1 shows the intrinsic densities obtained in these calculations. For ${ }^{9} \mathrm{Be}$, both the lowest-energy negative-parity solution and the excited positive-parity one are shown. In these figures, the $X$-, $Y$-, and $Z$-axes in the body-fixed frame 
EPJ Web of Conferences
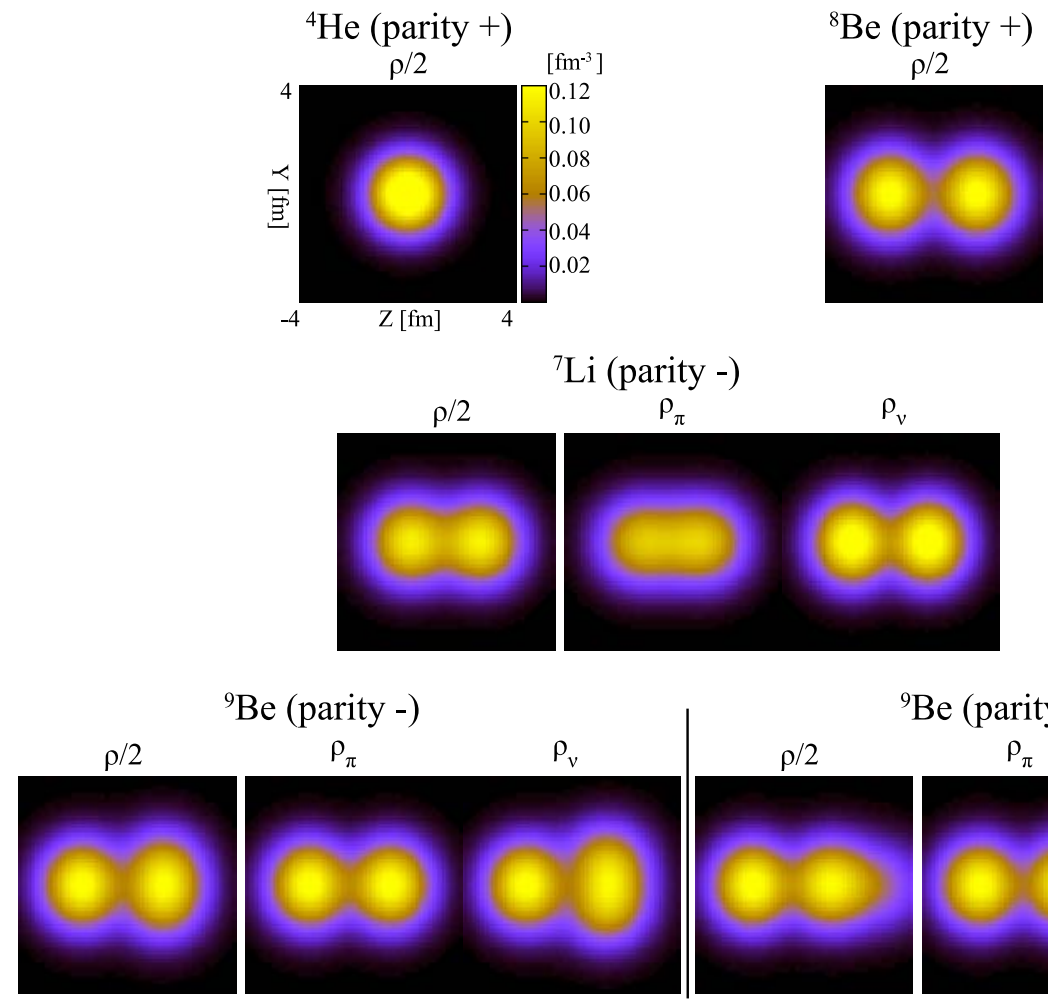

${ }^{9} \mathrm{Be}($ parity +$)$

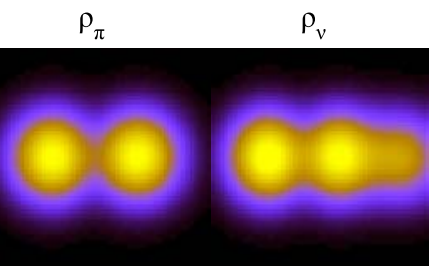

Fig. 1. Intrinsic density distributions of the lowest-energy parity solutions in ${ }^{4} \mathrm{He},{ }^{8} \mathrm{Be},{ }^{7} \mathrm{Li}$ and ${ }^{9} \mathrm{Be}$. In the matter $(\rho)$ density distributions, the densities are displayed as half values, and the proton $\left(\rho_{\pi}\right)$ and neutron $\left(\rho_{v}\right)$ density distributions are shown in the odd nuclei. The size of these squares is shown in the panel of ${ }^{4} \mathrm{He}$.

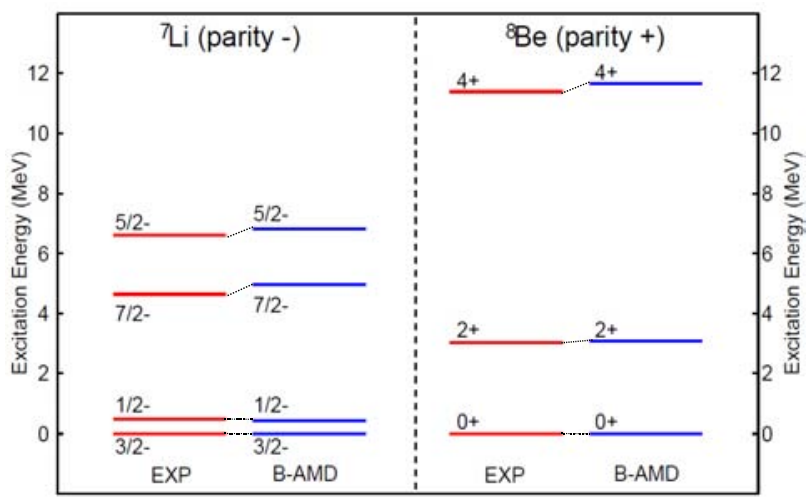

Fig. 2. Low-lying energy-level schemes of the experiments and Brueckner-AMD calculations labeled "EXP" and "B-AMD", respectively. The result of ${ }^{7} \mathrm{Li}$ is in the left panel and that of ${ }^{8} \mathrm{Be}$ is in the right panel. The obtained ground-state binding energies of ${ }^{7} \mathrm{Li}$ and ${ }^{8} \mathrm{Be}$ are $-29.6 \mathrm{MeV}$ and $-44.0 \mathrm{MeV}$, respectively.

are chosen so as to be $\left\langle\sum_{i} z_{i}^{2}\right\rangle \geq\left\langle\sum_{i} y_{i}^{2}\right\rangle \geq\left\langle\sum_{i} x_{i}^{2}\right\rangle$, and each density distribution in the $Z-Y$ plane is displayed. As seen in Fig. 1, the density of ${ }^{4} \mathrm{He}$ has a compact spherical shape. The density of ${ }^{8} \mathrm{Be}$ shows the well-known $\alpha+\alpha$ structure. In this result, the density of ${ }^{7} \mathrm{Li}$ do not present the explicit cluster structure as seen in an almost symmetric distribution. In the densities of ${ }^{9} \mathrm{Be}$, the structure of two $\alpha$ -

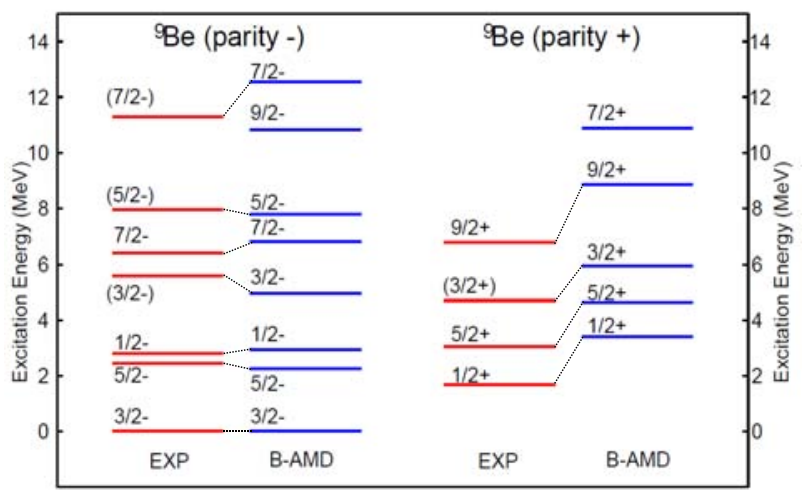

Fig. 3. Lowlying energy-level scheme of ${ }^{9} \mathrm{Be}$. The designations of the capital labels are explained in the caption of Fig. 2. The obtained ground-state binding energy is $-41.9 \mathrm{MeV}$.

clusters plus a valence neutron can be seen. In the negativeparity state of ${ }^{9} \mathrm{Be}$, the density distribution of the valence neutron corresponds to a $\pi$-orbit; on the other hand, that of the positive-parity state corresponds to a $\sigma$-orbit.

The calculated energy-level schemes of ${ }^{7} \mathrm{Li},{ }^{8} \mathrm{Be}$, and ${ }^{9}$ Be are shown in Figs. 2 and 3, respectively. The experimental energy level are compared with these results in 


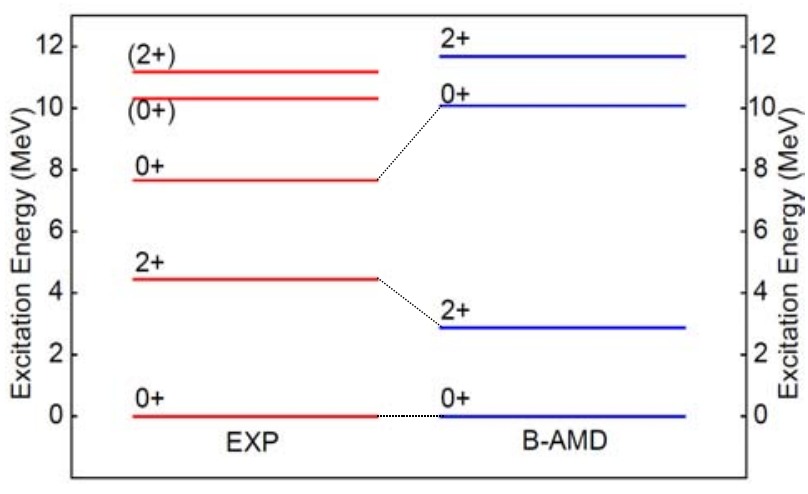

Fig. 4. The energy-level scheme of the positive-parity states with the excitation energy below $12 \mathrm{MeV}$ in ${ }^{12} \mathrm{C}$. The designations of the capital labels are explained in the caption of Fig. 2. The obtained ground-state binding energy is $-73.7 \mathrm{MeV}$.

these figures, where the groud states are normalized to zero energy. Each binding energy of the ground state is referred in each caption of the figure. The genuine three-body forces needed to reproduce the experimental binding energies are not considered in this framework. However, the obtained binding energy of the ground state $0^{+}$for ${ }^{4} \mathrm{He}$ is -24.6 $\mathrm{MeV}$ and its value is comparable to that obtained by fewbody calculations [7], $-25.9 \mathrm{MeV}$. The result of ${ }^{4} \mathrm{He}$ indicates the quantitative reliability of this calculation. In the result of ${ }^{8} \mathrm{Be}$, which is indicated to be the $\alpha+\alpha$ structure, the rotational band is reproduced well as seen in Fig. 2. Unfortunately, the result of the ground-state binding energy of ${ }^{8} \mathrm{Be}$ is underestimated to the double value of the binding energy of $\alpha$ particle, ${ }^{4} \mathrm{He}$. That indicates it is necessary to decribe the intercluster motion by using, for example, the generator coordinate method (GCM) [28] to reproduce the threshold of two $\alpha$ particles.

As seen in Figs. 2 and 3, this framework can provide the reasonal results for odd nuclei. In the result of ${ }^{7} \mathrm{Li}$, the energy levels are reproduced well. In the result of ${ }^{9} \mathrm{Be}$, the energy levels including the first excited states, $3 / 2_{2}^{-}, 5 / 2_{2}^{-}$, and $7 / 2_{2}^{-}$, which can be described by the $K$-mixing, are also reproduced well. In these calculations, in the negativeparity states of ${ }^{9} \mathrm{Be}$, the group of the dominant $K=3 / 2$ i.e. $K^{\pi}=3 / 2^{-}$band contains $3 / 2_{1}^{-}, 5 / 2_{1}^{-}$, and $7 / 2_{1}^{-}$states, while the $1 / 2_{1}^{-}, 3 / 2_{2}^{-}, 5 / 2_{2}^{-}$, and $7 / 2_{2}^{-}$states belong to the $K^{\pi}=1 / 2^{-}$band. The $9 / 2_{1}^{-}$state is described coherently by the $K=1 / 2$ and $K=3 / 2$ states, which has almost equal weights to the $K=1 / 2$ and $K=3 / 2$. The positive-parity states of ${ }^{9} \mathrm{Be}$ are described as the $K^{\pi}=1 / 2^{+}$band. Note that the results of the energy levels related to the spin-orbit splittig, for example, $3 / 2^{-}$and $1 / 2^{-}$in ${ }^{9} \mathrm{Be}$ are improved in comparison with our previous work [11] because the $G$ matrix includes the explicit spin-orbit term which generates the spin-orbit splittig in the energy level. The intervals of the energy levels in the positive parity states of ${ }^{9} \mathrm{Be}$ are reproduced well, however, the excitation energy of the low-

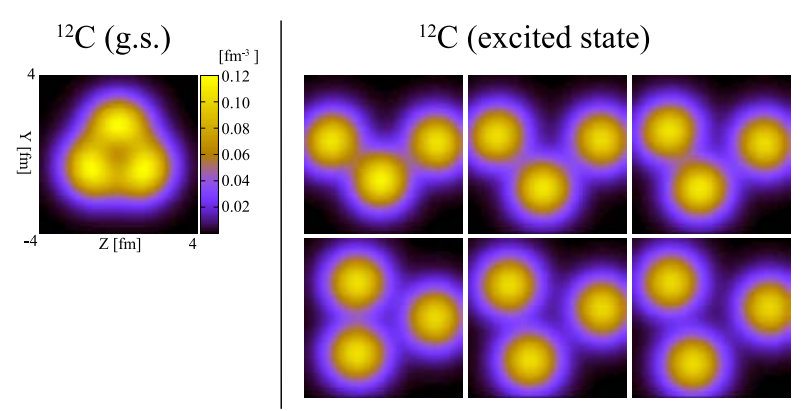

Fig. 5. The density distribution of the ground-state solution and typical ones included in the excited $0_{2}^{+}$solution labeled ${ }^{212} \mathrm{C}$ (g.s.)" and "12 C(excited state)", respectively. The configurations of the $0_{2}^{+}$state are calculated by using Eq. (44) with the root-mean-square mass radius $R=2.8-3.4$ (fm) and the approximated deformation parameter $\beta=0.49-0.76$ as the quantities under the constraint.

est positive-parity state $1 / 2^{+}$goes up from the result of our previous work [11] because the ground state $3 / 2_{1}^{-}$becomes more bound due to the spin-orbit force in the $G$-matrix.

\subsection{Description of the $0_{2}^{+}$state in ${ }^{12} \mathbf{C}$}

Here, we attempt to decribe not only the ground state $0^{+}$ but also the excited $\mathrm{O}_{2}^{+}$state in ${ }^{12} \mathrm{C}$ within the BruecknerAMD framework. It is known that the present shell-model approaches [21] have difficulty in describing the $0_{2}^{+}$states in ${ }^{12} \mathrm{C}$ because huge shell-model space is needed. On the other hand, cluster models [16-20] have been succeeded in describing this state. To describe higher $0^{+}$states in ${ }^{12} \mathrm{C}$, it is indicated that it is necessary to superpose a number of spatial configurations by the cluster models, for example, the GCM for $3 \alpha$ [16]. In this work, we creat various states without any assumption for configurations. For this aim, we apply the cooling method under the constraint $[14,22]$ :

$$
\frac{d \mathbf{Z}_{j}}{d t}=-\frac{\partial \mathcal{H}}{\partial \mathbf{Z}_{j}^{*}}-\sum_{k} \frac{\partial C^{k}}{\partial \mathbf{Z}_{j}^{*}}, \quad C^{k} \equiv\left(W_{k}-\left\langle W_{k}\right\rangle\right)^{2},
$$

where $W_{k}$ is the value of quantity under the constraint and $\left\langle\mathcal{W}_{k}\right\rangle$ represents its expectation value. In these calculations, we take the root-mean-square mass radius $R$ and the approximated deformation parameter $\beta$ [14] as the quantities under the constraint $W_{k}$. In addition, one should describe the configurations different from the ground state in viewpoint of the orthogonality of the excited state. Therefore, we perform the energy variation for the wave functions $\left|\Phi^{i}\right\rangle$ with the orthogonality to the ground state $\left|\Phi\left\{\boldsymbol{Z}^{0}\right\}\right\rangle$ as

$$
\left|\Phi^{i}\right\rangle=\left|\Phi\left\{\boldsymbol{Z}^{i}\right\}\right\rangle-\left|\Phi\left\{\boldsymbol{Z}^{0}\right\}\right\rangle \cdot \frac{\left\langle\Phi\left\{\boldsymbol{Z}^{0}\right\} \mid \Phi\left\{\boldsymbol{Z}^{i}\right\}\right\rangle}{\left\langle\Phi\left\{\boldsymbol{Z}^{0}\right\} \mid \Phi\left\{\boldsymbol{Z}^{0}\right\}\right\rangle},
$$


where $\{\boldsymbol{Z}\}$ represent the spatial configuration of the AMD wave packets as $\{\boldsymbol{Z}\} \equiv \boldsymbol{Z}_{1}, \boldsymbol{Z}_{2}, \cdots, \boldsymbol{Z}_{12}$, and determine the excited configurations $\left\{\boldsymbol{Z}^{i}\right\}$. In this work, we determine the ground state $\left|\Phi\left\{\boldsymbol{Z}^{0}\right\}\right\rangle$ and the excited states $\left|\Phi\left\{\boldsymbol{Z}^{i}\right\}\right\rangle$ as not the $J^{\pi}$-projected states but the parity-projected ones for simplicity of calculations, therefore we impose it on the energy variation that the inertial axes of each state are fitted to prevent us from obtaining the solutions of the rotated ground state: $\left\langle\sum_{i} z_{i}^{2}\right\rangle \geq\left\langle\sum_{i} y_{i}^{2}\right\rangle \geq\left\langle\sum_{i} x_{i}^{2}\right\rangle$. We use the Gaussian width parameter in Eq. (2), $v=0.195\left(\mathrm{fm}^{-2}\right)$, as the optimum value for $\left|\Phi\left\{Z^{0}\right\}\right\rangle$. Then we obtain the solutions $\left|\Phi_{n}^{J \pm}\right\rangle$ as the $J^{\pi}$-projected states,

$$
\left|\Phi_{n}^{J \pm}\right\rangle=\sum_{i=0} \sum_{K} C_{n}^{J \pm}(i, K)\left|\Phi_{M K}^{J \pm}\left\{\boldsymbol{Z}^{i}\right\}\right\rangle,
$$

where $\left|\Phi_{M K}^{J_{ \pm}^{ \pm}}\left\{\boldsymbol{Z}^{i}\right\}\right\rangle$ represents the $J^{\pi}$-projected state of $\left|\Phi\left\{\boldsymbol{Z}^{0}\right\}\right\rangle$ or $\left|\Phi\left\{\boldsymbol{Z}^{i}\right\}\right\rangle$, and the weights of the states $C_{n}^{J \pm}(i, K)$ are determined by diagonalizing $\left\langle\Phi_{M K}^{J^{ \pm}}\left\{\boldsymbol{Z}^{i}\right\}|\hat{H}| \Phi_{M K^{\prime}}^{J^{ \pm}}\left\{\boldsymbol{Z}^{i \prime}\right\}\right\rangle$ and $\left\langle\Phi_{M K}^{J_{ \pm}^{ \pm}}\left\{\boldsymbol{Z}^{i}\right\} \mid \Phi_{M K^{\prime}}^{J^{ \pm}}\left\{\boldsymbol{Z}^{i \prime}\right\}\right\rangle$ simultaneously.

In Fig. 4, the calculated energy-level scheme of the positive-partiy states in ${ }^{12} \mathrm{C}$ with the experimental data and the obtained intrinsic densities for the ground state and the excited $0_{2}^{+}$state are shown. We obtain the solution of the excited $0_{2}^{+}$state with the excitation energy $\sim 10 \mathrm{MeV}$. This excited $0_{2}^{+}$state contains of the explicit $3 \alpha$ configurations as seen in Fig. 5, which has no dominant $3 \alpha$ configuration because these states have almost equal weights in the $0_{2}^{+}$ state. This feature seems to be indicated as that of the $3 \alpha$ condensed state [20] which is the present interpretation of the $0_{2}^{+}$state in ${ }^{12} \mathrm{C}$. In the calculation with the condensed wave function [20], it is indicated that this $0_{2}^{+}$state has very large mass radius with $\sim 4 \mathrm{fm}$, therefore, we may need to superpose more states with a larger spread to improve the description of $\mathrm{O}_{2}^{+}$state in this framework. However, the $G$-matrix within this framework cannot be defined in the states with a large spread because the $\psi_{k l}^{0}$ in Eq. (15) becomes the scattering state and the $G$-matrix cannot be determined self-consistently due to the change of the boundary condition in the case that the single-particle energies become shallow and their $\omega$ in Eq. (17) becomes the positive value. In such a case, we may need to develop the methods to extrapolate the $G$-matrix. In addition, the $3 \alpha$ threshold energy is not reproduced in this work. Therefore, it is considered that it may be necessary to improve the ground and excited states simultaneously as the following works.

\section{Summary}

In this work, we developed the framework of AMD with realistic nuclear interactions based on the Brueckner theory, Brueckner-AMD. In the Brueckner-AMD, the $G$-matrix between the single-particle orbits in AMD can be determined from the realistic interactions self-consistently without any corrections. The correlation functions which are determined by the solutions of Bethe-Goldstone equation in the Brueckner theory and the model wave functions of a pair construct the $G$-matrix as the explicit effective interactions with non-central operators. In these calculations, we decribed the reasonable cluster structures and reproduced the low-lying energy levels of some light nuclei starting from the realistic nuclear force (AV8'). Futhermore, we decribed the $0_{2}^{+}$state in ${ }^{12} \mathrm{C}$, which is not solved sufficiently by the shell model approaches, within this framework starting from the realistic nuclear force.

\section{References}

1. R.B. Wiringa, S.C. Pieper, J. Carlson and V.R. Pandharipande, Phys. Rev. C62 (2000) 014001.

2. C. Forssén, E. Caurier and P. Navrátil, Phys. Rev. C79 (2009) 021303.

3. G. Hagen, T. Papenbrock, D.J. Dean, M. HjorthJensen and B.V. Asokan, Phys. Rev. C80 (2009) 021306.

4. T. Neff and H. Feldmeier, Nucl. Phys. A738, (2004) 357.

5. S. Fujii, T. Mizusaki, T Otsuka, T Sebe and A. Arima, Phys. Lett. B650 (2007) 9.

6. T. Myo, H. Toki and K. Ikeda, Prog. Theor. Phys. 121, (2009) 511.

7. H. Kamada et al., Phys. Rev. C64 (2001) 044001.

8. K. Ikeda, T. Marumori, R. Tamagaki and H. Tanaka, Prog. Theor. Phys. Suppl. No. 52, (1972) 1.

9. Y. Kanada-En'yo, H. Horiuchi and A. Ono, Phys. Rev. C52 (1995) 628

Y. Kanada-En'yo and H. Horiuchi,

Prog. Theor. Phys. Suppl. No.142, (2001) 205.

10. T. Togashi and K. Katō, Prog. Theor. Phys. 117, (2007) 189.

11. T. Togashi, T. Murakami and K. Katō, Prog. Theor. Phys. 121, (2009) 299.

12. T. Togashi, T. Murakami and K. Katō, Int. J. Mod. Phys. E17, (2008) 2081; J. Phys.: Conference Series 111, (2008) 012026.

T. Togashi, K. Katō and T. Murakami, Int. J. Mod. Phys. A24, (2009) 2142.

T. Murakami, T. Togashi and K. Katō, Mod. Phys. Lett. A24, (2009) 1013.

13. K. A. Brueckner, C.A. Levinson and H.M. Mahmoud, Phy. Rev. 95 (1954) 217.

K.A. Brueckner, Phy. Rev. 96 (1954) 508; Phy. Rev. 97 (1955) 1353.

K.A. Brueckner and J.L. Gammel, Phy. Rev. 109 (1958) 1023.

K.A. Brueckner and K.S. Masterson, Jr., Phy. Rev. 128 (1962) 2267.

H.A. Bethe, B.H. Brandow and A.G. Petschek, Phy. Rev. 129 (1963) 225.

H.A. Bethe, Phy. Rev. 138 (1965) B804.

14. A. Doté, Y. Kanada-En'yo and H. Horiuchi, Phys. Rev. C56 (1997) 1844.

15. H.A. Bethe, Phy. Rev. 103 (1956) 1353. J. Goldstone, Proc. R. Soc. A239, (1957) 267.

16. E. Uegaki, Y. Abe, S. Okabe and H. Tanaka, Prog. Theor. Phys. 62, (1979) 1621. 
$19^{\text {th }}$ International IUPAP Conference on Few-Body Problems in Physics

17. M. Kamimura, Nucl. Phys. A351, (1981) 456.

18. Y. Kanada-En'yo, Prog. Theor. Phys. 117, (2007) 655.

19. M. Chernykh, H. Feldmeier, T. Neff, P.von NeumannCosel and A. Richter, Phys. Rev. Lett. 98 (2007) 032501.

20. Y. Funaki, A. Tohsaki, H. Horiuchi, P. Schuck and G. Röpke, Eur. Phys. J. A24, (2005) 321.

21. P. Navrátil, J.P. Vary and B.R.Barrett, Phys. Rev. Lett. 84 (2000) 5728.

22. Y. Taniguchi, M. Kimura and H. Horiuchi, Prog. Theor. Phys. 112, (2004) 475.

23. M. Baldo, A. Fiasconaro, H.Q. Song, G. Giansiracusa and U. Lombardo, Phys. Rev. C65 (2001) 017303.

24. H. Bandō, S. Nagata and Y. Yamamoto,

Prog. Theor. Phys. 44, (1970) 646; Prog. Theor. Phys. 45, (1971) 1515.

25. S. Nagata, H. Bandō and Y. Akaishi,

Prog. Theor. Phys. Suppl. No. 65, (1979) 10.

26. L. Wilet, E.M. Henley, M. Kraft and A.D. MacKellar, Nucl. Phys. A282, (1977) 341.

H. Horiuchi, Nucl. Phys. A522, (1991) 257c.

A. Ono, H. Horiuchi, T. Maruyama and A. Ohnishi, Phys. Rev. Lett. 68 (1992) 2898; Prog. Theor. Phys. 87, (1992) 1185.

27. B.S. Pudliner, V.R. Pandharipande, J. Carlson, S.C. Pieper and R.B. Wiringa, Phys. Rev. C56 (1997) 1720.

R.B. Wiringa and S.C. Pieper, Phys. Rev. Lett. 89 (2002) 182501.

28. J.J. Griffin and J.A. Wheeler, Phy. Rev. 108 (1957) 311. 